\title{
FROM PRIMITIVE FORMS TO FROBENIUS MANIFOLDS
}

KYOJI SAITO AND ATSUSHI TAKAHASHI

\section{Contents}

1. Introduction 2

2. Flat structure and Frobenius Manifold Structure 3

2.1. Axioms 3

2.2. Flat Coordinates and Flat Potentials 4

2.3. Spectrum, Exponents and Weight 4

3. Universal Unfolding of Isolated Singularities 5

3.1. Universal Unfolding 5

3.2. Critical Set $\mathcal{C}$ and Product Structure on $\mathcal{T}_{S}$. 7

3.3. Primitive Vector Field and Euler Vector Field 7

3.4. Discriminant Locus and Milnor's Fibration Theorem 8

4. Filtered de Rham Cohomology Groups 8

4.1. $\quad$ Filtered de Rham Cohomology Group $\mathcal{H}_{F} \quad 8$

4.2. Gauß-Manin Connection 10

4.3. Degeneration of Hodge to de Rham 11

5. Higher Residue Pairings 12

5.1. Definition and Existence of Higher Residue Pairings 12

6. Primitive Forms 13

6.1. Definition and Existence of Primitive Forms 13

7. Construction of Frobenius Manifold Structures via Primitive Forms 15

7.1. Important Properties of Primitive Forms $\quad 15$

7.2. Identity Vector Field 16

7.3. Flatness of Gauß-Manin Connection 16

7.4. Flatness of the Higher Residue Parings 18

Appendix 18

References $\quad 20$ 


\section{INTRODUCTION}

In early 1980'th, the first named author, in the study of the period map associated with a primitive form [S3, S4], found certain differential geometric structure on the base space of a universal unfolding of an isolated hypersurface singularity. He called it the flat structure because that the structure contains a flat metric (bi-linear form) on the tangent bundle of the base space as its basic ingredient.

The finding of the flat structure was inspired from the same structure already found by the same author on the quotient variety of a finite reflection group [S1] [S-Y-S] where the invariant functions were called the flat invariants (c.f. [Du-Z],[S7],[S9],[Sat]). The introduction of these flat structures was motivated from the study of period map [S6], where one needs a particular coordinate system on the deformation parameter space, called the flat coordinates (see [S10] and its references for the classical examples).

The same structure was found by B. Dubrovin in his study of 2-dimensional topological field theories [Du] in order to study WDVV equations and their relation with integrable systems. The structure is axiomatized under the name of a Frobenius manifold structure because of the Frobenius ring structure on the tangent bundle (see [He1], [Ma1], [Sab]). In the present paper, for simplicity, we shall call a manifold with a flat structure, or, equivalently, a manifold with a Frobenius structure a Frobenius manifold.

As we observed, there are several constructions of Frobenius manifolds (c.f. [Ma1]). There are roughly three classes of geometric origins: invariant theories of Weyl groups, quantum cohomologies and deformation theories of complex varieties. Frobenius manifolds coming from different classes look, at a glance, different, however, they sometimes happen to coincide. Actually, mirror symmetry gives isomorphism between the Frobenius manifold from the quantum cohomology of a variety and that from the deformation theory of another variety. It is interesting to study the isomorphism since it provides unexpected information such as number of curves in the variety [CDGP].

By homological mirror symmetry conjecture by Kontsevich [Ko], these isomorphisms of Frobenius manifolds are expected to be derived from equivalences of triangulated (or $\left.A_{\infty^{-}}\right)$categories, in particular, it is expected that we can construct Frobenius manifolds from deformations of these categories in a unified way.

Although the original construction in [S3, S4] of the flat structure by the use of a primitive form is formulated only to the deformation space of germs of hypersurface isolated singularities, the construction itself, using the semi-infinite Hodge structure, is of general nature. It can be applied as the prototype to many other settings including the one in homological mirror symmetry, and has inspired several works on the semi-infinite Hodge theory ([G], [Ba], [Ba-Ko], [Do-Sab 1], [Do-Sab 2], [He2], [I], [Ka-Ko-P], [Ko-So]). 
Since this way of construction of the Frobenius manifold was not stated explicitly in the literature, the present paper tries to fill this lack of literatures. Namely, in section 2 of the present paper, we recall the axioms of the Frobenius manifold. In sections 3-6, we recall the construction and the definition of the primitive forms by a use of semi-infinite Hodge structure, and then in section 7 we show how a Frobenius manifold structure is deduced form a primitive form.

Acknowledgment. The authors express their gratitude to Ron Donagi for his invitation and support to contribute the present paper. This work is partly supported by Grant-in Aid for Scientific Research grant numbers 16340016, 17654015 and 17740036 from the Ministry of Education, Culture, Sports, Science and Technology, Japan.

\section{Flat structure and Frobenius Manifold Structure}

In the present section, we recall the axioms of the Frobenius manifold structure, i.e. that of the flat structure, on a manifold $M$. For simplicity, we shall call such $M$ a Frobenius manifold. Then, we state some of the immediate consequences of the axioms.

\subsection{Axioms. ([Du])}

Definition 2.1. Let $M=\left(M, \mathcal{O}_{M}\right)$ be a connected complex manifold of dimension $\mu$ whose holomorphic tangent sheaf is denoted by $\mathcal{T}_{M}$. A Frobenius manifold structure of rank $\mu$ on $M$ is a tuple $(\eta, \circ, e, E)$, where $\eta$ is a non-degenerate $\mathcal{O}_{M}$-symmetric bilinear form on $\mathcal{T}_{M}$, $\circ$ is $\mathcal{O}_{M}$-bilinear product on $\mathcal{T}_{M}$, defining an associative and commutative $\mathcal{O}_{M}$-algebra structure with the unit $e$, and $E$ is a holomorphic vector field on $M$, called the Euler vector field, which are subject to the following axioms:

- The product $\circ$ is self-adjoint with respect to $\eta$ : that is,

$$
\eta\left(\delta \circ \delta^{\prime}, \delta^{\prime \prime}\right)=\eta\left(\delta, \delta^{\prime} \circ \delta^{\prime \prime}\right), \quad{ }^{\forall} \delta, \delta^{\prime}, \delta^{\prime \prime} \in \mathcal{T}_{M},
$$

- The Levi-Civita connection $\nabla /: \mathcal{T}_{M} \rightarrow \mathcal{T}_{M} \otimes \Omega_{M}^{1}$ with respect to $\eta$ is flat: that is,

$$
\left[\nabla / \delta, \nabla / \delta_{\delta^{\prime}}\right]=\nabla /\left[\delta, \delta^{\prime}\right] \quad \forall \delta, \delta^{\prime} \in \mathcal{T}_{M}
$$

- The tensor $C: \mathcal{T}_{M} \rightarrow \operatorname{End}_{\mathcal{O}_{M}} \mathcal{T}_{M}$ defined by $C_{\delta} \delta^{\prime}:=\delta \circ \delta^{\prime}\left(\delta, \delta^{\prime} \in \mathcal{T}_{M}\right)$ is flat: that is,

$$
\nabla / C=0 .
$$

- The unit element $e$ of the o-algebra is a $\nabla /$-flat holomorphic vector field: that is,

$$
\nabla / e=0 .
$$


- The product $\circ$ and the metric $\eta$ are homogeneous of degree 1 and $2-d(d \in \mathbb{C})$, respectively, with respect to Lie derivative $L_{E}$ of Euler vector field $E$ : that is,

$$
\operatorname{Lie}_{E}(\mathrm{o})=\circ, \quad \operatorname{Lie}_{E}(\eta)=(2-d) \eta .
$$

\subsection{Flat Coordinates and Flat Potentials.}

Let us consider the space of horizontal sections of the connection $\nabla /$ :

$$
\mathcal{T}_{M}^{f}:=\left\{\delta \in \mathcal{T}_{M} \mid \nabla /{ }_{\delta^{\prime}} \delta=0{ }^{\forall} \delta^{\prime} \in \mathcal{T}_{M}\right\}
$$

which is, due to (2.2), a local system of rank $\mu$ on $M$ such that the metric $\eta$ takes constant values $\eta\left(\delta, \delta^{\prime}\right)$ for ${ }^{\forall} \delta, \delta^{\prime} \in \mathcal{T}_{M}^{f}$. Since $\mathcal{T}_{M}^{f}$ is abelian under the bracket product, we have:

Definition 2.2 (Flat coordinates). At each point of $M$, there exists a local coordinate system $\left(t_{0}, \ldots, t_{\mu-1}\right)$, called flat coordinates, such that

i) $e=\partial_{0}$ and ii) $\partial_{i}:=\frac{\partial}{\partial t_{i}}(i=0, \cdots, \mu-1)$ span $\mathcal{T}_{M}^{f}$ over $\mathbb{C}$,

Note 2.3. The vector field $e=\partial_{0}$ is sometimes called a primitive vector field, or a primitive derivation, in connection with the theory of primitive forms.

Let us state a consequence of the axiom $\nabla / C=0(2.3)$, implying the existence of the "potential" for the flat metric $\eta$ in the following sense:

Proposition 2.4 (Potential). At each point of $M$, there exists a local holomorphic function $\mathcal{F}$, called the flat potential, satisfying

$$
\eta\left(\partial_{i} \circ \partial_{j}, \partial_{k}\right)=\eta\left(\partial_{i}, \partial_{j} \circ \partial_{k}\right)=\partial_{i} \partial_{j} \partial_{k} \mathcal{F}, \quad{ }_{i, j}, k=0, \ldots, \mu-1,
$$

for any system of flat coordinates. In particular, one has

$$
\eta_{i j}:=\eta\left(\partial_{i}, \partial_{j}\right)=\partial_{0} \partial_{i} \partial_{j} F \text {. }
$$

\subsection{Spectrum, Exponents and Weight.}

Let $M$ be a Frobenius manifold of rank $\mu$. The gradient $\nabla / E$ of the Euler vector field $E$ is a tensor in $E n d_{\mathcal{O}_{M}} \mathcal{T}_{M}$. From Axioms, one sees easily that it is flat: $\nabla /(\nabla / E)=0$, and, hence, it induces a $\mathbb{C}$-endomorphism of the flat subspace $\mathcal{T}_{M}^{f}$. In the sequel of the present paper, we shall assume that the endomorphism is semi-simple. Let $1-q_{0}, \cdots, 1-q_{\mu-1}$ be the eigenvalues of the endomorphism at any point (independent of the point due to the connectivity of $M$ ), where we normalize $q_{0}=0$ since $\nabla /{ }_{e} E=e$. By using suitable flat coordinates $\left(t^{0}, \cdots, t^{\mu-1}\right)$ such that $\nabla /{ }_{\partial_{i}} E=\left(1-q_{i}\right) \partial_{i}$, the Euler vector filed is expressed as $E=\sum_{i=0}^{\mu-1}\left\{\left(1-q_{i}\right) t^{i}+r_{i}\right\} \partial_{i}$, for suitable constants $r_{i}$ depending on the choice of the center of the flat coordinates. 
Definition 2.5 (Spectrum, Weight and Exponents). Suppose $\nabla / E$ is semi-simple.

(i) The set $\left\{q_{0}, q_{1}, \ldots, q_{\mu-1}\right\}$ is called the spectrum.

(ii) Let us fix a non-negative integer $w \in \mathbb{Z}_{\geq 0}$, called a weight of the manifold $M$. Depending on a choice of a weight $w$, we define the minimal exponent by $r:=$ $(w-d) / 2 \in \mathbb{C}$ and the set of exponents by the eigenvalues $r+q_{0}, \cdots, r+q_{\mu-1}$ of the endomorphism $N:=-\nabla / E+(r+1) \cdot \mathrm{id}_{\mathcal{T}_{M}}$ (see section 7 for the meaning of $r$ and $N)$.

\section{Universal Unfolding of Isolated Singularities}

In the following sections 3-6, we introduce the concept of a primitive form.

In the present section, we define a universal unfolding of an isolated singularity and give its basic properties. As we shall see, the concept of a universal unfolding already induces an algebra structure on the tangent bundle of its base space and a concept of the Euler vector field on it. The fiber dimension of the unfolding shall give the weight in the definition of the exponents in Definition 2.4. However, the minimal exponent and the flat metric will be introduced later in section 6 depending on a choice of a primitive form.

\subsection{Universal Unfolding.}

Let $f(\underline{x})=f\left(x_{0}, \cdots, x_{n}\right)$ be a holomorphic function defined on a neighborhood of the origin in $\mathbb{C}^{n+1}$ with $f(0)=0$. Assume that

$$
\mathcal{O}_{\mathbb{C}^{n+1}, 0} /\left(\frac{\partial f}{\partial x_{0}}, \ldots, \frac{\partial f}{\partial x_{n}}\right)
$$

is of finite rank, say $\mu$, over $\mathbb{C}$. This is equivalent to that the function $f$ defined on $\mathbb{C}^{n+1}, 0$ has an isolated critical point at the origin 0 , or equivalently, the germ of the hyper surface $X_{0}:=\{f=0\} \subset\left(\mathbb{C}^{n+1}, 0\right)$ at the origin has at most an isolated singular point at the origin.

The universal unfolding of $f$ is an extension $F$ of $f$ defined on $\mathbb{C}^{n+1} \times \mathbb{C}^{\mu}$ extended by $\mu$ dimensional parameter $\underline{t} \in \mathbb{C}^{\mu}$ defined as follows, where we denote by

$$
p: \mathbb{C}^{n+1} \times \mathbb{C}^{\mu} \longrightarrow \mathbb{C}^{\mu},(\underline{x}, \underline{t}) \mapsto \underline{t}
$$

the projection map from the total space to the parameter space.

Definition 3.1 ( R. Thom [Th]). A holomorphic function $F$ defined on a neighborhood $\mathcal{X}$ of the origin $(0,0)$ of $\mathbb{C}^{n+1} \times \mathbb{C}^{\mu}$ is called a universal unfolding of $f$, if it satisfies:

(i) $\left.F\right|_{p^{-1}(0)}=f$ in a neighborhood of the origin in $p^{-1}(0) \simeq \mathbb{C}^{n+1}$.

(ii) There exists an $\mathcal{O}_{S, 0}$-isomorphism

$$
\mathcal{T}_{\mathbb{C}^{\mu}, 0} \rightarrow p_{*} \mathcal{O}_{\mathbb{C}^{n+1} \times \mathbb{C}^{\mu}, 0} /\left(\frac{\partial F}{\partial x_{0}}, \ldots, \frac{\partial F}{\partial x_{n}}\right), \quad \delta \mapsto \widehat{\delta} F
$$


where $\widehat{\delta}$ is a lifting on $\mathbb{C}^{n+1} \times \mathbb{C}^{\mu}$ of a vector field $\delta$ with respect to the projection $p$.

Fact 3.2. For any germ of holomorphic function $f$ defining an isolated singularity at the origin, there exists a universal unfolding.

Proof. Choose a system of holomorphic functions $1=: \phi_{0}(\underline{x}), \phi_{1}(\underline{x}), \ldots, \phi_{\mu-1}(\underline{x})$ forms a $\mathbb{C}$-linear basis of $\mathcal{O}_{\mathbb{C}^{n+1}, 0} /\left(\frac{\partial f}{\partial x_{0}}, \ldots, \frac{\partial f}{\partial x_{n}}\right)$ and set a function

$$
F(\underline{x}, \underline{t}):=f(\underline{x})+\sum_{i=0}^{\mu-1} t_{i} \cdot \phi_{i}(\underline{x}),
$$

defined in a neighborhood of $(0,0)$, where $\left(t_{0}, \ldots, t_{\mu-1}\right)$ is a coordinate system of $\mathbb{C}^{\mu}$.

Let $F(\underline{x}, \underline{t})$ be a universal unfolding of $f$. By fixing a domain $\mathcal{X}$ and a range $\mathbb{C}_{\delta}$ of $F$ and a (small) open set $S \subset \mathbb{C}^{\mu}$ of the unfolding parameters $\underline{t} \in \mathbb{C}^{\mu}$, we consider the map $\varphi=(F, p): \mathcal{X} \rightarrow \mathbb{C}_{\delta} \times S$, which defines a flat family of hyper surfaces where the fiber over 0 is isomorphic to the singularity $X_{0}:=\{f=0\}$ and generic fibers are somethings of $X_{0}$ containing vanishing cycles to the singularity. This is achieved as follows. Let us fix Euclidean norms $\|\cdot\|$ on $\mathbb{C}^{n+1} \times \mathbb{C}^{\mu}, \mathbb{C}$ and $\mathbb{C}^{\mu}$. For positive real numbers $r, \delta$ and $\epsilon$, put

$$
\begin{aligned}
S_{\epsilon} & :=\left\{\underline{t} \in \mathbb{C}^{\mu} \mid\|\underline{t}\|<\epsilon\right\} \\
\mathbb{C}_{\delta} & :=\{w \in \mathbb{C} \mid\|w\|<\delta\} \\
X_{r, \delta, \epsilon} & :=\left\{(\underline{x}, \underline{t}) \in \mathbb{C}^{n+1} \times \mathbb{C}^{\mu} \mid\|(\underline{x}, \underline{t})\|<r,\|F(\underline{x}, \underline{t})\|<\delta\right\} \cap p^{-1}\left(S_{\epsilon}\right)
\end{aligned}
$$

For the choice $1 \gg r \gg \delta \gg \epsilon>0$ of the radius, we have

(i) $p: X_{r, \delta, \epsilon} \rightarrow S_{\epsilon}$ is a smooth Stein map, which is topologically acyclic.

(ii) The holomorphic map

$$
\varphi: X_{r, \delta, \epsilon} \longrightarrow \mathbb{C}_{\delta} \times S_{\epsilon}, \quad(\underline{x}, \underline{t}) \mapsto(w, \underline{t}):=(F(\underline{x}, \underline{t}), \underline{t})
$$

is a flat Stein map, whose fibers are smooth and transversely to $\partial X_{r, \delta . \epsilon}$ at each point of $\partial X_{r, \delta . \epsilon}$. The fiber $\varphi^{-1}(0) \cap X_{r, \delta . \epsilon}$ over 0 has only an isolated singular point isomorphic to $X_{0}$ and is contractible to a point.

We study some basic properties of the family $\varphi(3.5)$ in the following $3.2,3.3$ and 3.4. At each step and also at the study of de Rham cohomology groups in section 4, we need to choose the constants $r, \delta$ and $\epsilon$ suitably. Since it is cumbersome to state about the choice at each time, we fix the constants once for all and put $\mathcal{X}:=X_{r, \delta, \varepsilon}$ and $S:=S_{\varepsilon}$. 


\subsection{Critical Set $\mathcal{C}$ and Product Structure on $\mathcal{T}_{S}$.}

Let the setting be as in 3.1. Let us denote by $\mathcal{C}$ the critical set of the family (3.5), or, equivalent to say, the relative critical set of $F$ with respect to the projection $p$. That is, $\mathcal{C}$ is the support of the sheaf:

$$
\mathcal{O}_{\mathcal{C}}:=\mathcal{O}_{\mathcal{X}} /\left(\frac{\partial F}{\partial x_{0}}, \ldots, \frac{\partial F}{\partial x_{n}}\right)
$$

The assumption that $f$ has an isolated singularity implies $\mathcal{C} \cap p^{-1}(0)=\{(0,0)\}$, and, hence, $\left.p\right|_{\mathcal{C}}$ is a proper finite map. Actually, $\frac{\partial F}{\partial x_{0}}, \ldots, \frac{\partial F}{\partial x_{n}}$ form a regular sequence at the origin and $\mathcal{C}$ is Cohen-Macaulay of dimension equal to that of $S$ so that $\mathcal{C} \rightarrow S$ is a flat finite cover. Therefore, $p_{*} \mathcal{O}_{\mathcal{C}}$ is a locally free sheaf on $S$ of rank $\mu$.

The universality (ii) of the unfolding $F$, implies the the following $\mathcal{O}_{S}$-isomorphism:

$$
\mathcal{T}_{S} \simeq p_{*} \mathcal{O}_{\mathcal{C}},\left.\quad \delta \mapsto \widehat{\delta} F\right|_{\mathcal{C}}
$$

By this isomorphism, the tangent bundle $\mathcal{T}_{S}$ naturally obtains an $\mathcal{O}_{S}$-algebra structure.

Definition 3.3 (Product on $\mathcal{T}_{S}$ ). We shall denote by o the induced product structure on $\mathcal{T}_{S}$. Namely, for $\delta, \delta^{\prime} \in \mathcal{T}_{S}$, we have

$$
\left.\widehat{\left(\delta \circ \delta^{\prime}\right.}\right)\left.F\right|_{\mathcal{C}}:=\left.\left.\widehat{\delta} F\right|_{\mathcal{C}} \cdot \widehat{\delta^{\prime}} F\right|_{\mathcal{C}}
$$

o is called the residual product.

Note 3.4. Originally $[\mathrm{S} 2,3,4,5]$, the product o was denoted by $*$ and called the $*$-product.

Note 3.5. Consider the complex $\left(\wedge^{\bullet} \mathcal{T}_{\mathcal{X} / S}, d F \wedge\right)$ of $\mathcal{O}_{\mathcal{X}}$-modules with $\mathcal{O}_{\mathcal{X}}$ in degree 0 :

$$
0 \longrightarrow \wedge^{n+1} \mathcal{T}_{\mathcal{X} / S} \stackrel{d F \wedge}{\longrightarrow} \wedge^{n} \mathcal{T}_{\mathcal{X} / S} \stackrel{d F \wedge}{\longrightarrow} \cdots \stackrel{d F \wedge}{\longrightarrow} \mathcal{T}_{\mathcal{X} / S} \stackrel{d F \wedge}{\longrightarrow} \mathcal{O}_{\mathcal{X}} \longrightarrow 0 .
$$

Since $\frac{\partial F}{\partial x_{0}}, \ldots, \frac{\partial F}{\partial x_{n}}$ form a regular sequence, the complex (3.9) is quasi-isomorphic to

$$
p_{*} \mathcal{O}_{\mathcal{X}} / p_{*}\left(d F \wedge \mathcal{T}_{\mathcal{X} / S}\right) \simeq p_{*} \mathcal{O}_{\mathcal{C}}
$$

\subsection{Primitive Vector Field and Euler Vector Field.}

Through the isomorphism (3.7), we introduce two particular vector fields on $S$.

Definition 3.6. The vector field $\delta_{0}$ on $S$ corresponding to the unit $1 \in \mathcal{O}_{\mathcal{C}}$ is called the primitive vector field. That is, $\left.\widehat{\delta_{0}} F\right|_{\mathcal{C}}=1$. The vector field $E$ on $S$ corresponding to $\left.F\right|_{\mathcal{C}} \in \mathcal{O}_{\mathcal{C}}$ is called the Euler vector field. That is, $\left.(\widehat{E} F)\right|_{\mathcal{C}}=\left.F\right|_{\mathcal{C}}$. 


\subsection{Discriminant Locus and Milnor's Fibration Theorem.}

The image of the finite map $\left.\varphi\right|_{\mathcal{C}}$ is a one-codimensional analytic subvariety (i.e. a divisor) in $\mathbb{C}_{\delta} \times S$, which we shall denote by $\mathcal{D}$ and call the discriminant locus of the unfolding $F$. The following is due to J. Milnor and H. Hamm (c.f. [Mi]):

Theorem 3.7. The restriction $\left.\varphi\right|_{\mathcal{X} \backslash \varphi^{-1}(\mathcal{D})}$ is a locally trivial fibration, whose general fiber $\varphi^{-1}(t)$ for $t \in S \backslash \mathcal{D}$ is homotopic to a bouquet of $\mu$ copies of $n$-sphere $S^{n}$.

Corollary 3.8. Fix a point $\left(w_{0}, t\right) \in \partial \mathbb{C}_{\delta} \times S$ and set $\mathcal{X}_{t}:=p^{-1}(\underline{t})$ and $B_{t}:=\varphi^{-1}\left(w_{0}, t\right)$. Then one has

$$
H_{n+1}\left(\mathcal{X}_{t}, B_{t} ; \mathbb{Z}\right) \simeq H_{n}\left(B_{t} ; \mathbb{Z}\right) \simeq \mathbb{Z}^{\mu}
$$

This leads us to study relative de Rham cohmology groups of the family $(F, p: \mathcal{X} \rightarrow S)$ as the residues of the forms on $\mathcal{X}$, and, hence, to a primitive form.

\section{Filtered de Rham Cohomology Groups}

We study the relative de Rham cohmology group of the family $p: \mathcal{X} \rightarrow S$ in a non-classical manner. That is, compared with the classical de Rham theory, we introduce an auxiliary formal variable $\delta_{w}$. This variable was necessarily introduced in [S5] in order to define the higher residue pairings (see section 5.) on the filtered de Rham cohomology groups. Due to this new variable, the study of the classical Hodge theory switches to the study of the semi-infinite Hodge theory (c.f. Givental [G], Barannikov [Ba], BarannikovKontsevich [Ba-Ko], Hertling [He2], Iritani [I], Katzarkov-Kontsevich-Pantev [Ka-Ko-P], Kontsevich-Soibelman [Ko-So], and others).

\subsection{Filtered de Rham Cohomology Group $\mathcal{H}_{F}$.}

Let the setting $(F, p: \mathcal{X} \rightarrow S)$ be as in section 3. Let us consider the complex:

$$
\left(\Omega_{\mathcal{X} / S}^{\bullet}\left[\left[\delta_{w}^{-1}\right]\right]\left[\delta_{w}\right], \delta_{w}^{-1} d_{\mathcal{X} / S}+d F \wedge\right)
$$

of sheaves on $\mathcal{X}$, where $d_{\mathcal{X} / S}$ is the classical de Rham differential relative to the morphism $p: \mathcal{X} \rightarrow S, \delta_{w}$ is a formal variable which commutes with the wedge product and with the differentiation $d_{\mathcal{X} / S}$, and we set $\Omega_{\mathcal{X} / S}^{i}\left[\left[\delta_{w}^{-1}\right]\right]\left[\delta_{w}\right]:=\Omega_{\mathcal{X} / S}^{i} \otimes_{\mathcal{O}_{S}} \mathcal{O}_{S}\left[\left[\delta_{w}^{-1}\right]\right]\left[\delta_{w}\right]$.

Since $p$ is a Stein map and the singularities of the family $\varphi$ are isolated, the direct image $\mathbb{R} p_{*}$ of (4.1) is pure $n+1$-dimensional, that is, its only non-vanishing cohomology group is

$$
\mathbb{R}^{n+1} p_{*}\left(\Omega_{\mathcal{X} / S}^{\bullet}\left[\left[\delta_{w}^{-1}\right]\right]\left[\delta_{w}\right], \delta_{w}^{-1} d+d F \wedge\right) .
$$


Definition 4.1. We set

$$
\mathcal{H}_{F}:=\mathbb{R}^{n+1} p_{*}\left(\Omega_{\mathcal{X} / S}^{\bullet}\left[\left[\delta_{w}^{-1}\right]\right]\left[\delta_{w}\right], \delta_{w}^{-1} d_{\mathcal{X} / S}+d F \wedge\right)
$$

and call it the filtered de Rham cohomology group of the universal unfolding $(F, p: \mathcal{X} \rightarrow$ $S)$.

The direct image $\mathbb{R} p_{*}$ of the truncation $\left(\Omega_{\mathcal{X} / S}^{\bullet}\left[\left[\delta_{w}^{-1}\right]\right] \delta_{w}^{-k}, \delta_{w}^{-1} d+d F \wedge\right)$ of the complex (4.1) for $k \in \mathbb{Z}$ is again pure $n+1$-dimensional and its cohomology group

$$
\mathcal{H}_{F}^{(-k)} \simeq \mathbb{R}^{n+1} p_{*}\left(\Omega_{\mathcal{X} / S}^{\bullet}\left[\left[\delta_{w}^{-1}\right]\right] \delta_{w}^{-k}, \delta_{w}^{-1} d+d F \wedge\right)
$$

is a $\mathcal{O}_{S}\left[\left[\delta_{w}^{-1}\right]\right]$-free module of rank $\mu$. One can show further that they are naturally submodules of $\mathcal{H}_{F}$ so that they form an increasing and exhaustive filtration of $\mathcal{H}_{F}$

$$
\begin{gathered}
\cdots \subset \mathcal{H}_{F}^{(-i-1)} \subset \mathcal{H}_{F}^{(-i)} \subset \cdots \subset \mathcal{H}_{F}^{(-1)} \subset \mathcal{H}_{F}^{(0)} \subset \cdots \subset \mathcal{H}_{F}, \\
\bigcup_{i \in \mathbb{Z}} \mathcal{H}_{F}^{(-i)}=\mathcal{H}_{F} \quad \text { and } \bigcap_{i \in \mathbb{Z}} \mathcal{H}_{F}^{(-i)}=\{0\}
\end{gathered}
$$

such that $\mathcal{H}_{F}$ is complete with respect to the filtration.

The multiplication of $\delta_{w}$ induces an $\mathcal{O}_{S^{-}}$isomorphism

$$
\delta_{w}: \mathcal{H}_{F}^{(-i)} \simeq \mathcal{H}_{F}^{(-i+1)} .
$$

The $k$ th graded piece $\operatorname{gr}^{(k)}\left(\mathcal{H}_{F}\right):=\mathcal{H}_{F}^{(-k)} / \mathcal{H}_{F}^{(-k-1)}$ of the filtration (4.5) is isomorphic to

$$
\Omega_{F}:=p_{*} \Omega_{\mathcal{X} / S}^{n+1} / p_{*}\left(d F \wedge \Omega_{\mathcal{X} / S}^{n}\right)
$$

by a homomorphism $r^{(k)}:=r^{(0)} \circ \delta_{w}^{k}$, where $r^{(0)}$ is given by the natural short exact sequence:

$$
0 \rightarrow \mathcal{H}_{F}^{(-1)} \subset \mathcal{H}_{F}^{(0)} \stackrel{r^{(0)}}{\longrightarrow} \Omega_{F} \rightarrow 0
$$

Note 4.2. Let $\pi$ is the natural projection: $\mathbb{C} \times S \rightarrow S$. The $\mathcal{O}_{S}\left\{\delta_{w}^{-1}\right\}$-free module $\mathcal{H}_{0}:=\pi_{*}\left(\mathbb{R}^{n} \varphi_{*}\left(\Omega_{\mathcal{X} / \mathbb{C}_{\delta} \times S}\right)\right)$ was first considered by Brieskorn [B], where he further introduced two $\mathcal{O}_{S}\left\{\delta_{w}^{-1}\right\}$-free modules $\mathcal{H}_{0}^{\prime}:=\pi_{*}\left(\varphi_{*}\left(\Omega_{\mathcal{X} / \mathbb{C}_{\delta} \times S}^{n}\right) / d\left(\varphi_{*}\left(\Omega_{\mathcal{X} / \mathbb{C}_{\delta} \times S}^{n-1}\right)\right)\right)$ and $\mathcal{H}_{0}^{\prime \prime}:=$ $\pi_{*}\left(\varphi_{*}\left(\Omega_{\mathcal{X} / \mathbb{C}_{\delta} \times S}^{n+1}\right) / d F \wedge d\left(\varphi_{*}\left(\Omega_{\mathcal{X} / \mathbb{C}_{\delta} \times S}^{n-1}\right)\right)\right)$ such that

$$
\mathcal{H}_{0} \subset \mathcal{H}_{0}^{\prime} \subset \mathcal{H}_{0}^{\prime \prime}
$$

where $\mathcal{H}_{0}^{\prime \prime}$ is known as the Brieskorn lattice. 
We have the isomorphisms of the Brieskorn lattices with the filters of the filtered de Rham cohomology groups as follows (see [S5] 3.)

$$
\begin{aligned}
\mathcal{H}_{0} \otimes_{\mathcal{O}_{S}\left\{\delta_{w}^{-1}\right\}} \mathcal{O}_{S}\left[\left[\delta_{w}^{-1}\right]\right] & \simeq \mathcal{H}_{F}^{(-2)} \\
\mathcal{H}_{0}^{\prime} \otimes_{\mathcal{O}_{S}\left\{\delta_{w}^{-1}\right\}} \mathcal{O}_{S}\left[\left[\delta_{w}^{-1}\right]\right] & \simeq \mathcal{H}_{F}^{(-1)} \\
\mathcal{H}_{0}^{\prime \prime} \otimes_{\mathcal{O}_{S}\left\{\delta_{w}^{-1}\right\}} \mathcal{O}_{S}\left[\left[\delta_{w}^{-1}\right]\right] & \simeq \mathcal{H}_{F}^{(0)}
\end{aligned}
$$

where we set $\mathcal{O}_{S}\left\{\delta_{w}^{-1}\right\}:=\mathcal{O}_{S} \otimes_{\mathbb{C}} \mathbb{C}\left\{\delta_{w}^{-1}\right\}$ and $\mathbb{C}\left\{\delta_{w}^{-1}\right\}$ is the ring of convergent power series in $\delta_{w}^{-1}$. This gives the geometric significance to the filtered cohomology group $\mathcal{H}_{F}$ (as the de Rham cohmology group of the Milnor fibration (3.3)).

Like $\mathcal{H}_{F}^{(0)}, \mathcal{H}_{F}^{(-1)}$ and $\mathcal{H}_{F}^{(-2)}$, every $\mathcal{O}_{S}\left[\left[\delta_{w}^{-1}\right]\right]$-modules treated in this paper are completions of some $\mathcal{O}_{S}\left\{\delta_{w}^{-1}\right\}$-modules, This holomorphicity plays an important role when we shall construct primitive forms in section 6 .

Note 4.3. Comparing (3.10) with (4.8), we observe that $\Omega_{C}$ is a $p_{*} \mathcal{O}_{\mathbb{C}}$ module of rank 1 . That is, by choosing a form $\zeta \in \Gamma\left(\mathcal{X}, \Omega_{\mathcal{X} / S}^{n+1}\right)$, we have $\mathcal{O}_{S}$-isomorphisms of three modules:

$$
\mathcal{T}_{S} \simeq p_{*} \mathcal{O}_{\mathcal{C}} \simeq \Omega_{F},\left.\left.\quad \delta \mapsto \widehat{\delta} F\right|_{\mathcal{C}} \mapsto \widehat{\delta} F\right|_{\mathcal{C}} \zeta
$$

where the last isomorphism in (4.10) depends on the class of $\zeta$ in $\Omega_{F}$. This means that the $\mathcal{O}_{S}$-module $\mathcal{T}_{S}$ is equipped with the three structures:

(i) Lie algebra structure on $\mathcal{T}_{S}$ with the unit vector field and Euler vector field,

(ii) associative commutative algebra structure coming from that on $p_{*} \mathcal{O}_{\mathcal{C}}$,

(iii) non-degenerate $\mathcal{O}_{S}$-symmetric bilinear pairing coming from the residue pairing on $\Omega_{F}$ (c.f. [Ha]):

$$
\eta_{F}\left(\omega_{1}, \omega_{2}\right):=\operatorname{Res} \mathcal{X} / S\left[\begin{array}{c}
\phi_{1} \phi_{2} d x_{0} \wedge \cdots \wedge d x_{n} \\
\frac{\partial F}{\partial x_{0}} \cdots \frac{\partial F}{\partial x_{n}}
\end{array}\right]
$$

for the classes of $\omega_{1}=\phi_{1} d x_{0} \wedge \cdots \wedge d x_{n}$ and $\omega_{2}=\phi_{2} d x_{0} \wedge \cdots \wedge d x_{n}$ in $\Omega_{F}$.

These are the first approximation of the flat structure on $\mathcal{T}_{S}$ (i.e., we have a Frobenius "algebra" structure). In order to assert the integrability of metric $\eta$, we need to choose and lift the class of $\zeta$ in $\Omega_{F}$ to a good object in $\mathcal{H}_{F}^{(0)}$, which is the primitive form, introduced in section 6 .

\subsection{Gauß-Manin Connection.}

Likewise classical de Rham cohomology groups, we introduce the Gauss-Manin connection on the filtered de Rham cohomology group $\mathcal{H}_{F}$, where the covariant differentiation 
is defined not only for the vector fields on $S$ but also for the derivation of the formal variable $\delta_{w}{ }^{1}$. Namely, let us consider the following $\mathcal{O}_{S}\left[\left[\delta_{w}^{-1}\right]\right]$ free-module of rank $\mu+1$ :

$$
\mathcal{T}_{\mathbb{A}_{\delta_{w}^{1}}^{1} \times S}:=\mathcal{O}_{S}\left[\left[\delta_{w}^{-1}\right]\right] \frac{d}{d \delta_{w}} \oplus \mathcal{O}_{S}\left[\left[\delta_{w}^{-1}\right]\right] \otimes_{\mathcal{O}_{S}} \mathcal{T}_{S}
$$

which naturally carries the Lie algebra structure by extending that on $\mathcal{T}_{S}$ and by letting $\frac{d}{d \delta_{w}}$ commute with $\mathcal{T}_{S}$, where we note that $\frac{d}{d \delta_{w}} \delta_{w}^{-1}=-\delta_{w}^{-2}$.

Definition 4.4. We define a connection, which we call again the Gauß-Manin connection,

$$
\nabla: \mathcal{T}_{\mathbb{A}^{1} \delta_{w}^{-1}} \times S=\mathcal{H}_{F} \rightarrow \mathcal{H}_{F}
$$

by letting, for $\delta \in \mathcal{T}_{S}$ and $\zeta=\left[\phi d x_{0} \wedge \cdots \wedge d x_{n}\right] \in \mathcal{H}_{F}$,

$$
\begin{aligned}
& \nabla_{\frac{d}{d \delta_{w}}} \zeta:=\left[\left(F(\underline{x}, \underline{t}) \phi+\frac{d \phi}{d \delta_{w}}\right) d x_{0} \wedge \cdots \wedge d x_{n}\right], \\
& \nabla_{\delta} \zeta:=\delta_{w}\left[\left(\delta_{w}^{-1} \delta \phi+\delta F \phi\right) d x_{0} \wedge \cdots \wedge d x_{n}\right] .
\end{aligned}
$$

From the definition, we immediately have the followings:

Proposition 4.5. (i) The Gauss-Manin connection is integrable:

$$
\left[\nabla_{\delta}, \nabla_{\delta^{\prime}}\right]=\nabla_{\left[\delta, \delta^{\prime}\right]}, \quad{ }^{\forall} \delta, \delta^{\prime} \in \mathcal{T}_{\mathbb{A}_{\delta_{w}^{1}}^{1} \times S} .
$$

(ii) The Gauss-Manin connection satisfies the following transversality condition:

$$
\nabla: \mathcal{T}_{S} \otimes \mathcal{H}_{F}^{(-k-1)} \longrightarrow \mathcal{H}_{F}^{(-k)}
$$

(iii) The covariant differentiation $\nabla_{\frac{d}{d \delta_{w}}}$ preserves the filters

$$
\nabla_{\frac{d}{d \delta_{w}}}\left(\mathcal{H}_{F}^{(-k)}\right) \subset \mathcal{H}_{F}^{(-k)}
$$

This implies, $\nabla_{\frac{d}{d \delta_{w}^{-1}}}=-\delta_{w}^{2} \nabla_{\frac{d}{d \delta_{w}}}$ has a pole of order 2 at $\delta_{w}^{-1}=0$.

\subsection{Degeneration of Hodge to de Rham.}

It will be very important to consider $\mathcal{H}_{F}^{(i)}$ as a deformation of a sheaf on the formal line $\mathbb{A}_{\delta_{w}^{-1}}^{1}$ over the parameter space $S$ when we shall construct a primitive form. By the Corollary 3.8 of Milnor's fibration theorem, we have the following:

Proposition 4.6. $\mathcal{H}_{F}^{(i)}$ is flat over $\mathcal{O}_{S}\left[\left[\delta_{w}^{-1}\right]\right]$. In particular, at each point $t \in S,\left.\mathcal{H}_{F}^{(i)}\right|_{t}$ gives a vector bundle of rank $\mu$ over $\mathbb{A}_{\delta_{w}^{-1}}^{1}$ whose generic fiber is isomorphic to $H^{n+1}\left(\mathcal{X}_{t}, B_{t}\right)$ and the fiber at $\delta_{w}^{-1}=0$ is isomorphic to $H^{n}\left(B_{t}\right)$.

\footnotetext{
${ }^{1}$ In the original formulation $[\mathrm{S} 3, \mathrm{~S} 4]$, the covariant differentiation with respect to the variable $\delta_{w}$ was not explicitly used, but implicitly by the use of the formula (4.14a) and the higher residue pairings (see section 5, [S5]). In the present paper, we make the use of that covariant differentiation explicit.
} 
Note 4.7. This seems to be one of the motivation to formulate the Hodge to de Rham degeneration conjecture in the semi-infinite Hodge theory for Calabi-Yau $A_{\infty}$-categories (c.f. $[\mathrm{Ko}-\mathrm{So}])$.

\section{Higher Residue Pairings}

In this section, we claim an existence of a non-degenerate $\mathcal{O}_{S}\left[\left[\delta_{w}^{-1}\right]\right]\left[\delta_{w}\right]$ pairing on the filtered de Rham cohomology group $\mathcal{H}_{F}$, which we call the higher residue pairing, since it extends the classical residue pairing (4.11) to the infinite sequences.

\subsection{Definition and Existence of Higher Residue Pairings.}

Let $(F, p: \mathcal{X} \rightarrow S)$ be as in section 3 the data of a universal unfolding of $f$, and let $\mathcal{H}_{F}$ be its filtered de Rham cohomolgy group given in section 4 . To state the result, let us fix a notation: for $P=\sum_{k \in \mathbb{Z}} p_{k} \delta_{w}^{k} \in \mathcal{O}_{S}\left[\left[\delta_{w}^{-1}\right]\right]\left[\delta_{w}\right]$, define $P^{*}:=\sum_{k \in \mathbb{Z}} p_{k}\left(-\delta_{w}\right)^{k}$ such that $P=\left(P^{*}\right)^{*}$.

Theorem 5.1 (K. Saito [S5]). There exists a unique, up to a constant factor, $\mathcal{O}_{S}$-bilinear form:

$$
K_{F}: \mathcal{H}_{F} \times \mathcal{H}_{F} \rightarrow \mathcal{O}_{S}\left[\left[\delta_{w}^{-1}\right]\right]\left[\delta_{w}\right]
$$

satisfying the following properties:

(i) For all $\omega_{1}, \omega_{2} \in \mathcal{H}_{F}$,

$$
K_{F}\left(\omega_{1}, \omega_{2}\right)=K_{F}\left(\omega_{2}, \omega_{1}\right)^{*} .
$$

(ii) For any $P \in \mathcal{O}_{S}\left[\left[\delta_{w}^{-1}\right]\right]\left[\delta_{w}\right]$ and for all $\omega_{1}, \omega_{2} \in \mathcal{H}_{F}$,

$$
P K_{F}\left(\omega_{1}, \omega_{2}\right)=K_{F}\left(P \omega_{1}, \omega_{2}\right)=K_{F}\left(\omega_{1}, P^{*} \omega_{2}\right) .
$$

(iii) The following diagram is commutative:

$$
\begin{aligned}
K_{F}: \mathcal{H}_{F}^{(0)} \times \mathcal{H}_{F}^{(0)} & \longrightarrow & \delta_{w}^{-n-1} \mathcal{O}_{S}\left[\left[\delta_{w}^{-1}\right]\right] \\
r^{(0)} \times r^{(0)} \downarrow & & \downarrow \bmod \delta_{w}^{-n-2} \mathcal{O}_{S}\left[\left[\delta_{w}^{-1}\right]\right] \\
\eta_{F}: \Omega_{F} \times \Omega_{F} & \longrightarrow & \mathcal{O}_{S},
\end{aligned}
$$

(iv) For all $\omega_{1}, \omega_{2} \in \mathcal{H}_{F}$,

$$
\frac{d}{d \delta_{w}} K_{F}\left(\omega_{1}, \omega_{2}\right)=K_{F}\left(\nabla_{\frac{d}{d \delta_{w}}} \omega_{1}, \omega_{2}\right)+K_{F}\left(\omega_{1}, \nabla_{\frac{d}{d \delta_{w}}} \omega_{2}\right) .
$$

(v) For all $\omega_{1}, \omega_{2} \in \mathcal{H}_{F}$ and for any $\delta \in \mathcal{T}_{S}$,

$$
\delta K\left(\omega_{1}, \omega_{2}\right)=K\left(\nabla_{\delta} \omega_{1}, \omega_{2}\right)+K\left(\omega_{1}, \nabla_{\delta} \omega_{2}\right) .
$$


We define $K_{F}^{(k)}$ for $k \in \mathbb{Z}$ by the $k$-th coefficient of the expansion in $\delta_{w}$ :

$$
K_{F}\left(\omega_{1}, \omega_{2}\right):=\sum_{k} K_{F}^{(k)}\left(\omega_{1}, \omega_{2}\right) \delta_{w}^{-n-1-k}
$$

and call it the $k$-th higher residue pairing.

Remark 5.2. The conditions (K3) and (K4) together are equivalent to say that $K_{F}$ is flat with respect to the Gauß-Manin connection $\nabla$.

\section{Primitive Forms}

In the present section, we introduce the concept of a primitive form and show its local existence. Let $(F, p: \mathcal{X} \rightarrow S)$ be a universal unfolding of $f$.

\subsection{Definition and Existence of Primitive Forms.}

For an element $\zeta^{(0)} \in \Gamma\left(S, \mathcal{H}_{F}^{(0)}\right)$ and $k \in \mathbb{Z}$, we put $\zeta^{(-k)}:=\left(\delta_{w}\right)^{-k} \zeta^{(0)} \in \Gamma\left(S, \mathcal{H}_{F}^{(-k)}\right)$.

Definition 6.1 (K. Saito $[\mathrm{S} 3, \mathrm{~S} 4])$. An element $\zeta^{(0)} \in \Gamma\left(S, \mathcal{H}_{F}^{(0)}\right)$ is called a primitive form if it satisfies the following five conditions:

(i) $\zeta^{(0)}$ induces an $\mathcal{O}_{S}$-isomorphism

$$
\mathcal{T}_{S} \otimes_{\mathcal{O}_{S}} \mathcal{O}_{S}\left[\left[\delta_{w}^{-1}\right]\right] \simeq \mathcal{H}_{F}^{(0)}, \quad \sum_{k=0}^{\infty} \delta_{k} \cdot \delta_{w}^{-k} \mapsto \sum_{k=0}^{\infty} \nabla_{\delta_{k}} \zeta^{(-1)} \cdot \delta_{w}^{-k}
$$

(ii) For $k \geq 1$ and for all $\delta, \delta^{\prime} \in \mathcal{T}_{S}$,

$$
K^{(k)}\left(\nabla_{\delta} \zeta^{(-1)}, \nabla_{\delta^{\prime}} \zeta^{(-1)}\right)=0 .
$$

(iii) There exists a constant $r \in \mathbb{C}$ such that

$$
\nabla_{\frac{d}{d \delta_{w}}} \zeta^{(0)}=\nabla_{E} \zeta^{(-1)}-r \zeta^{(-1)} .
$$

(iv) For $k \geq 2$ and for all $\delta, \delta^{\prime}, \delta^{\prime \prime} \in \mathcal{T}_{S}$,

$$
K^{(k)}\left(\nabla_{\delta} \nabla_{\delta^{\prime}} \zeta^{(-2)}, \nabla_{\delta^{\prime \prime}} \zeta^{(-1)}\right)=0 .
$$

(v) For $k \geq 2$ and for all $\delta, \delta^{\prime} \in \mathcal{T}_{S}$,

$$
K^{(k)}\left(\nabla_{\frac{d}{d \delta_{w}}} \nabla_{\delta} \zeta^{(-1)}, \nabla_{\delta^{\prime}} \zeta^{(-1)}\right)=0
$$

Theorem 6.2 (M. Saito [SM]). There exists a primitive form $\zeta^{(0)}$.

The construction of a primitive form $\zeta^{(0)}$ consists of two steps. The first step is to show the existence of "a primitive form at the origin". Let us denote by $\mathcal{H}_{f}^{(0)}, \Omega_{f}, K_{f}$ and etc. the restrictions of $\mathcal{H}_{F}^{(0)}, \Omega_{F}, K_{F}$ and etc. to $0 \in S$. 
Lemma 6.3. One has a basis $\left\{v_{0}, \ldots, v_{\mu-1}\right\}$ of $\mathcal{H}_{f}^{(0)}$ over $\mathbb{C}\left[\left[\delta_{w}^{-1}\right]\right]$ satisfying the following conditions:

(i) The class $\left[v_{0}\right] \in \Omega_{f}$ of $v_{0}$ generates $\Omega_{f}$ over $\left.p_{*} \mathcal{O}_{\mathcal{C}}\right|_{\underline{t}=0}$.

(ii) The values $K_{f}\left(v_{i}, v_{j}\right)$ for $i, j=0, \cdots, \mu-1$ belongs to $\mathbb{C} \delta_{w}^{-n-1}$.

(iii) There exist matrices $C_{f} \in M(\mu, \mathbb{C})$ and $N_{0} \in M(\mu, \mathbb{Q})$ with $N_{0}$ diagonal, whose eigenvalues $\left\{\alpha_{0}, \ldots, \alpha_{\mu-1}\right\}$ of $N_{0}$ coincides with the exponents of the singularity $f$, such that

$$
\nabla_{\frac{d}{d \delta_{w}}} \vec{v}=C_{f} \vec{v}-\delta_{w}^{-1} N_{0} \vec{v}
$$

where $\vec{v}:={ }^{t}\left(v_{0}, \ldots, v_{\mu-1}\right)$.

Remark 6.4. In this first step, one should choose and fix exponents of $f$, which is one of most delicate issue in the construction of a primitive form.

Note 6.5. In order to prove the above Lemma, we have to solve the Riemann-HilbertBirkhoff problem: we have to show the existence of a flat vector bundle $H_{f}$ of rank $\mu$ over $\mathbb{P}_{\delta_{w}}^{1}:=\mathbb{A}_{\delta_{w}^{-1}}^{1} \cup\left\{\delta_{w}=0\right\}=\mathbb{A}_{\delta_{w}^{-1}}^{1} \cup \mathbb{A}_{\delta_{w}}^{1}$ such that $\mathcal{O}\left(\left.H_{f}\right|_{\mathbb{A}_{\delta_{w}^{-1}}^{1}}\right) \simeq \mathcal{H}_{f}^{(0)}$ and $\mathcal{O}\left(\left.H_{f}\right|_{\mathbb{A}_{\delta_{w}}^{1}}\right)$ has logarithmic poles along $\delta_{w}=0$, i.e., $\nabla_{d / d \delta_{w}} \mathcal{O}\left(\left.H_{f}\right|_{\mathbb{A}_{\delta_{w}}^{1}}\right) \subset \delta_{w}^{-1} \mathcal{O}\left(\left.H_{f}\right|_{\mathbb{A}_{\delta_{w}}^{1}}\right)$. This is done by choosing a good opposite filtration $S$ to $\mathcal{H}_{f}^{(0)}$, that is, a subspace $S \subset \mathcal{H}_{f}$ satisfying $\mathcal{H}_{f}=\mathcal{H}_{f}^{(0)} \oplus S, \delta_{w} S \subset S, K_{f}(S, S) \subset \delta_{w}^{-n+1} \mathbb{C}\left[\delta_{w}\right]$ and $\nabla_{d / d \delta_{w}} S \subset \delta_{w}^{-1} S$. Then, the vector space spanned by $\left\{v_{0}, \ldots, v_{\mu-1}\right\}$ is obtained as the intersection $\mathcal{H}_{f}^{(0)} \cap \delta_{w}^{-1} S$. The notion of the above opposite filtration is implicitly used in [SM], and is now the basic of the construction of primitive forms for general settings (c.f. [Ba], [He1], [Do-Sab 1], [Do-Sab 2], [Sab])

The second step is to extend the above basis $\left\{v_{0}, \ldots, v_{\mu-1}\right\}$ of $\mathcal{H}_{f}^{(0)}$ over $\mathbb{C}\left[\left[\delta_{w}^{-1}\right]\right]$ to a basis of $\mathcal{H}_{F}^{(0)}$ over $\mathcal{O}_{S}\left[\left[\delta_{w}^{-1}\right]\right]$. One needs to show the following Lemma:

Lemma 6.6. One has a basis $\left\{\zeta_{0}, \ldots, \zeta_{\mu-1}\right\}$ of $\mathcal{H}_{F}^{(0)}$ over $\mathcal{O}_{S}\left[\left[\delta_{w}^{-1}\right]\right]$ such that

(i) $\left.\zeta_{i}\right|_{\underline{t}=0}=v_{i}$ for all $i=0, \ldots, \mu-1$.

(ii) There exists a matrix $C_{F} \in M\left(\mu, \Gamma\left(S, \mathcal{O}_{S}\right)\right)$ with $\left.C_{F}\right|_{t=0}=C_{f}$ such that

$$
\nabla_{\frac{d}{d \delta_{w}}} \vec{\zeta}=C_{F} \vec{\zeta}-\delta_{w}^{-1} N_{0} \vec{\zeta}
$$

where $\vec{\zeta}:={ }^{t}\left(\zeta_{0}, \ldots, \zeta_{\mu-1}\right)$.

(iii) For any $\delta \in \mathcal{T}_{S}$, there exists a matrix $C_{\delta} \in M\left(\mu, \Gamma\left(S, \mathcal{O}_{S}\right)\right)$ such that

$$
\delta_{w}^{-1} \nabla_{\delta} \vec{\zeta}=C_{\delta} \vec{\zeta}
$$


Once we have the above two Lemmas, we obtain a primitive form $\zeta^{(0)}$ as $\zeta_{0}$.

Remark 6.7. For a given universal unfolding of an isolated singularity, the primitive form may not unique (up to a constant factor). For instance, simply elliptic singularity admits a one-parameter family of primitive forms ([S3]). It is still an open question to determine the space of all primitive forms for a given universal unfolding. The above construction depends on a choice of (the phases) of exponents, where some "wild" choice of exponents is still allowed. One expects that certain "tamed" class of primitive forms are to be constructed through an (infinite dimensional) Lie algebras, and some works are in progress ([K-S-T 1, K-S-T 2, K-S-T 3],[S8],[T1, T2]).

\section{Construction of Frobenius Manifold Structures via Primitive Forms}

Let $(F, p: \mathcal{X} \rightarrow S)$ be a universal unfolding of $f$. In the present section, we construct a Frobenius manifold structure on $S$ depending on a choice of a primitive form $\zeta^{(0)}$.

First, we give some direct consequences of the notion of primitive forms.

\subsection{Important Properties of Primitive Forms.}

Let us fix a primitive form $\zeta^{(0)}$. Note that, by $(\mathrm{P} 1)$,

$$
\mathcal{T}_{S} \simeq \Omega_{F} \simeq p_{*} \mathcal{O}_{\mathcal{C}},\left.\left.\quad \delta \mapsto \widehat{\delta} F\right|_{\mathcal{C}} \cdot r^{(0)}\left(\zeta^{(0)}\right) \mapsto \widehat{\delta} F\right|_{\mathcal{C}}
$$

is an $\mathcal{O}_{S}$-isomorphism.

Proposition 7.1. The bilinear form (again denoted by $\eta_{F}$ ) on $\mathcal{T}_{S}$ defined by

$$
\eta_{F}: \mathcal{T}_{S} \times \mathcal{T}_{S} \rightarrow \mathcal{O}_{S}, \quad\left(\delta, \delta^{\prime}\right) \mapsto \eta_{F}\left(r^{(0)}\left(\nabla_{\delta} \zeta^{(-1)}\right), r^{(0)}\left(\nabla_{\delta^{\prime}} \zeta^{(-1)}\right)\right)
$$

is symmetric and nondegenerate.

The property (P2) implies the following:

Proposition 7.2. For $\delta, \delta^{\prime} \in \mathcal{T}_{S}$, we have

$$
K_{F}\left(\nabla_{\delta} \zeta^{(-1)}, \nabla_{\delta^{\prime}} \zeta^{(-1)}\right) \in \delta_{w}^{-n-1} \mathcal{O}_{S}
$$

Moreover, the conditions (P1), (P2) and (P4) imply the following:

Proposition 7.3. There exists a connection $\nabla /: \mathcal{T}_{S} \times \mathcal{T}_{S} \rightarrow \mathcal{T}_{S}$ such that

$$
\nabla_{\delta} \nabla_{\delta^{\prime}} \zeta^{(-2)}=\nabla_{\delta \circ \delta^{\prime}} \zeta^{(-1)}+\nabla_{\nabla_{\delta} \delta^{\prime}} \zeta^{(-2)} .
$$

Similarly, the conditions (P1), (P2) and (P5) imply the following: 
Proposition 7.4. There exists an $\mathcal{O}_{S}$-endomorphism $N: \mathcal{T}_{S} \rightarrow \mathcal{T}_{S}$ such that

$$
\nabla_{\frac{d}{d \delta_{w}}} \nabla_{\delta} \zeta^{(-1)}=\nabla_{E \circ \delta} \zeta^{(-1)}-\nabla_{N \delta} \zeta^{(-2)}, \quad \delta \in \mathcal{T}_{S}
$$

Therefore, we obtain the following the main result of the present paper:

Theorem 7.5. Let $(F, p: \mathcal{X} \rightarrow S)$ be a universal unfolding of $f$. Choose a primitive form $\zeta^{(0)}$. Then $\left(\eta_{F}, \circ, \delta_{0}, E\right)$ is a Frobenius manifold structure of rank $\mu$ with dimension $d=n+1-2 r$.

The proof is an easy consequences of Propositions 7.1-7.4 together with flatness of higher residue pairings with respect to $\nabla$. It is the direct consequence of the abstract notion of higher residue parings and primitive forms, we write down the proof of Theorem down to the earth.

\subsection{Identity Vector Field.}

Set $\delta^{\prime}=\delta_{0}$ in (S2) and comparing it with (S2). Then, we obtain the following properties:

Proposition 7.6 (Flatness of the Identity).

$$
\nabla / \delta_{0}=0 \text {. }
$$

Proposition 7.7 (Homogeneity of the Identity).

$$
N\left(\delta_{0}\right)=r \cdot \delta_{0} .
$$

\subsection{Flatness of Gauß-Manin Connection.}

Flatness of Gauß-Manin connection $\nabla$ together with equations (S2) imply the following Lemma:

Lemma 7.8. For $\delta, \delta^{\prime}, \delta^{\prime \prime} \in \mathcal{T}_{S}$, we have

$$
\begin{aligned}
& {\left[\nabla_{\delta}, \nabla_{\delta^{\prime}}\right] \nabla_{\delta^{\prime \prime}} \zeta^{(0)}=\nabla_{\left[\delta, \delta^{\prime}\right]} \nabla_{\delta^{\prime \prime}} \zeta^{(0)}} \\
& \qquad\left\{\begin{array}{l}
\delta \circ\left(\delta^{\prime} \circ \delta^{\prime \prime}\right)-\delta^{\prime} \circ\left(\delta \circ \delta^{\prime \prime}\right)=0 \\
\nabla / \delta\left(\delta^{\prime} \circ \delta^{\prime \prime}\right)+\delta \circ\left(\nabla / \delta_{\delta^{\prime}} \delta^{\prime \prime}\right)-\nabla / \delta_{\delta^{\prime}}\left(\delta \circ \delta^{\prime \prime}\right)-\delta^{\prime} \circ\left(\nabla / \delta \delta^{\prime \prime}\right)=\left[\delta, \delta^{\prime}\right] \circ \delta^{\prime \prime} \\
\nabla / \delta\left(\nabla / \delta_{\delta^{\prime}} \delta^{\prime \prime}\right)-\nabla / \delta_{\delta^{\prime}}\left(\nabla / \delta \delta^{\prime \prime}\right)=\nabla /\left[\delta, \delta^{\prime}\right] \\
\delta^{\prime \prime}
\end{array}\right.
\end{aligned}
$$


By this Lemma, we have the following three consequences:

Proposition 7.9 (Connection $\nabla /$ is Flat and Torsion Free).

$$
\begin{gathered}
{\left[\nabla /{ }_{\delta}, \nabla / \delta_{\delta^{\prime}}\right]=\nabla /\left[\delta, \delta^{\prime}\right], \quad \delta, \delta^{\prime} \in \mathcal{T}_{S},} \\
\nabla / \delta_{\delta} \delta^{\prime}-\nabla / /_{\delta^{\prime}} \delta=\left[\delta, \delta^{\prime}\right], \quad \delta, \delta^{\prime} \in \mathcal{T}_{S} .
\end{gathered}
$$

Proposition 7.10 (Product $\circ$ is Potential).

$$
\nabla / C=0,
$$

where we denote by $C$ the $\mathcal{O}_{\mathcal{T}_{S}}$-linear map $C: \mathcal{T}_{S} \rightarrow \mathcal{T}_{S} \otimes \Omega_{S}^{1}$ defined by $C_{\delta} \delta^{\prime}:=\delta \circ \delta^{\prime}$, $\delta, \delta^{\prime} \in \mathcal{T}_{S}$.

Proposition 7.11 (Product $\circ$ is of degree 1).

$$
\operatorname{Lie}_{E}(\circ)=\circ,
$$

namely,

$$
\left[E, \delta \circ \delta^{\prime}\right]-[E, \delta] \circ \delta^{\prime}-\delta \circ\left[E, \delta^{\prime}\right]=\delta \circ \delta^{\prime}, \quad \delta, \delta^{\prime} \in \mathcal{T}_{S}
$$

On the other hand, the flatness of Gauß-Manin connection $\nabla$ together with equations (S3) imply the following Lemma:

Lemma 7.12. For $\delta, \delta^{\prime} \in \mathcal{T}_{S}$, we have

$$
\begin{aligned}
{\left[\nabla_{\delta}, \nabla_{\frac{d}{d \delta_{w}}}\right] \nabla_{\delta^{\prime}} \zeta^{(0)} } & =0 \\
& \Longleftrightarrow\left\{\begin{array}{l}
E \circ\left(\delta \circ \delta^{\prime}\right)=\delta \circ\left(E \circ \delta^{\prime}\right) \\
N\left(\delta \circ \delta^{\prime}\right)+\nabla /{ }_{\delta}\left(E \circ \delta^{\prime}\right)=E \circ \nabla /{ }_{\delta} \delta^{\prime}+\delta \circ N\left(\delta^{\prime}\right)+\delta \circ \delta^{\prime} \\
\nabla / \delta
\end{array}\right)
\end{aligned}
$$

By this Lemma, we have the following two consequences:

Lemma 7.13. For $\delta \in \mathcal{T}_{S}$, we have

$$
N(\delta)=-\nabla /{ }_{\delta} E+(r+1) \delta .
$$


Proposition 7.14 (Gradient of the Euler Vector Field $\nabla / E$ is Flat).

$$
\nabla / N=0 .
$$

\subsection{Flatness of the Higher Residue Parings.}

The property (S2) with the flatness $\nabla K_{F}=0$ imply the following two:

Proposition 7.15 (Product $\circ$ is $\eta_{F}$-invariant).

$$
\eta_{F}\left(\delta \circ \delta^{\prime}, \delta^{\prime \prime}\right)=\eta_{F}\left(\delta, \delta^{\prime} \circ \delta^{\prime \prime}\right), \quad \delta, \delta^{\prime}, \delta^{\prime \prime} \in \mathcal{T}_{S},
$$

Proposition 7.16 (Connection $\nabla /$ is metric).

$$
\delta \eta_{F}\left(\delta^{\prime}, \delta^{\prime \prime}\right)=\eta_{F}\left(\nabla / \delta_{\delta} \delta^{\prime}, \delta^{\prime \prime}\right)+\eta_{F}\left(\delta^{\prime}, \nabla / \delta_{\delta} \delta^{\prime \prime}\right), \quad \delta, \delta^{\prime}, \delta^{\prime \prime} \in \mathcal{T}_{S},
$$

The property (S3) with the flatness $d / d \delta_{w} K_{F}=0$ imply the following:

\section{Lemma 7.17 ( $\eta_{F}$ is homogeneous).}

$$
(n+1) \eta_{F}\left(\delta, \delta^{\prime}\right)=\eta_{F}\left(N(\delta), \delta^{\prime}\right)+\eta_{F}\left(\delta, N\left(\delta^{\prime}\right)\right), \quad \delta, \delta^{\prime} \in \mathcal{T}_{S}
$$

Combining this with Lemma 7.13, we have

Corollary $7.18(d=n+1-2 r)$.

$$
\operatorname{Lie}_{E}\left(\eta_{F}\right)=(2-(n+1-2 r)) \eta_{F} .
$$

We have verified all axioms of Frobenius manifold.

\section{APPENDIX}

It is expected from homological mirror symmetry conjecture that one can construct Frobenius manifolds from deformations of Calabi-Yau $A_{\infty}$-categories in a unified way. As is already written in Introduction, although the original construction, explained in this paper, of the Frobenius manifolds based on filtered de Rham cohomology groups, higher residue pairings and primitive forms is formulated only to universal unfoldings of isolated hypersurface singularities, several notion and the idea of this construction is of general 
nature. It has inspired and motivated the semi-infinite Hodge theory for Calabi-Yau $A_{\infty}$ categories (c.f. [Ka-Ko-P], [Ko-So]).

We shall give a list in which readers can find the way how objects in the original construction will be generalized and rewritten in this categorical language.

\begin{tabular}{|c|c|c|}
\hline objects in this paper & generalization & \\
\hline$\left(f, \mathbb{C}^{n+1}\right)$ & $\mathcal{A}$ & $\begin{array}{c}A_{\infty} \text {-category } \\
\text { (compact, smooth, Calabi-Yau) }\end{array}$ \\
\hline$J_{f}$ & $H H^{\bullet}(\mathcal{A}, \mathcal{A})$ & Hochschild cohomology \\
\hline$\left(\Omega_{\mathbb{C}^{n+1}}^{\bullet}, d f \wedge\right)$ & $\left(C_{\bullet}(\mathcal{A}, \mathcal{A}), b\right)$ & Hochschild chain complex \\
\hline$\Omega_{f}$ & $H H_{\bullet}(\mathcal{A}, \mathcal{A})$ & Hochschild homology \\
\hline$d$ & $B$ & Conne's differential \\
\hline$\delta_{w}^{-1}$ & $u$ & parameter of degree 2 \\
\hline $\mathcal{H}_{f}$ & $H P \bullet(\mathcal{A})$ & periodic cyclic homology \\
\hline $\mathcal{H}_{f}^{(0)}$ & $H C_{\bullet}^{-}(\mathcal{A})$ & negative cyclic homology \\
\hline $\begin{array}{l}\Omega_{f}\left[\left[\delta_{w}^{-1}\right]\right] \\
\Rightarrow \mathcal{H}_{f}^{(0)}\end{array}$ & $\begin{array}{l}H H_{\bullet}(\mathcal{A}, \mathcal{A})[[u]] \\
\quad \Rightarrow H C_{\bullet}^{-}(\mathcal{A})\end{array}$ & $\begin{array}{l}\text { Hodge to de Rham } \\
\text { spectral sequence }\end{array}$ \\
\hline$\frac{d}{d \delta_{w}}$ & $-u^{2} \frac{d}{d u}$ & $\begin{array}{l}\text { flat connection on } H C_{\bullet}^{-}(\mathcal{A}) \\
\text { with poles of order } 2 \text { at } u=0\end{array}$ \\
\hline$K_{f}$ & $?$ & higher residue pairings \\
\hline$J_{f} \stackrel{\zeta}{\simeq} \Omega_{f}$ & $\begin{array}{l}H H^{\bullet}(\mathcal{A}, \mathcal{A}) \\
\stackrel{\zeta}{\simeq} H H_{\bullet}(\mathcal{A}, \mathcal{A})\end{array}$ & $\begin{array}{c}\text { primitive form } \\
\text { (at the origin } 0 \in S \text { ) }\end{array}$ \\
\hline
\end{tabular}

If one takes $\mathcal{A}$ in the list as the dg enhancement of the category of singularity, then one recovers all objects given in the left column. As a result, the way of constructing Frobenius manifolds for isolated hypersurface singularities and that of Barannikov-Kontsevich [Ba] [Ba-Ko] for Calabi-Yau manifolds are unified. 


\section{REFERENCES}

[Ba] S. Barannikov, Quantum periods. I. Semi-infinite variations of Hodge structures, Internat. Math. Res. Notices 2001, 23, 1243-1264.

[Ba-Ko] S. Barannikov and M. Kontsevich, Frobenius manifolds and formality of Lie algebras of polyvector fields, Internat. Math. Res. Notices 1998, no. 4, 201-215.

[Br] T. Bridgeland, Stability conditions on triangulated category, math.AG/0212237.

[B] E. Brieskorn, Die Monodromie der isolierten Singularitäten von Hyperflächen, Manuscripta Math. 2 (1970), 103-161.

[CDGP] P. Candelas, X. de la Ossa, P. Green and L. Parkes, A pair of Calabi-Yau man-ifolds as an exactly soluble superconformal theory, Nucl. Phys. B 359 (1991) 21-74.

[Do-Sab 1] A. Douai and C. Sabbah, Gauss-Manin systems, Brieskorn lattices and Frobenius structures. I, Proceedings of the International Conference in Honor of Frederic Pham (Nice, 2002). Ann. Inst. Fourier (Grenoble) 53 (2003), no. 4, 1055-1116.

[Do-Sab 2] — Gauss-Manin systems, Brieskorn lattices and Frobenius structures. II, Frobenius manifolds, 1-18, Aspects Math., E36, Vieweg, Wiesbaden, 2004.

[Du] B. Dubrovin, Geometry of 2D Topological Field Theories, in Integrable system and Quantum Groups, Montecatini,Terme 1993. Lecture Note in Math. 1620, Springer-Verlag 1996, 120-348.

[Du-Z] B. Dubrovin and Y. Zhang, Extended affine Weyl groups and Frobenius manifolds, Compositio Math. 111 (1998), no. 2, 167-219.

[G] A. Givental, Gromov-Witten invariants and quantization of quadratic Hamiltonians, Mosc. Math. J. 1 (2001), no. 4, 551-568, 645.

[Ha] R. Hartshorn, Residue and Duality Lecture Notes in Mathematics, No. 20 Springer-Verlag, BerlinNew York 1966

[He1] C. Hertling, Frobenius manifolds and moduli spaces for singularities, Cambridge University Press, 2002.

[He2] C. Hertling, $t t^{*}$ geometry, Frobenius manifolds, their connections, and the construction for singularities, J. Reine Angew. Math. 555 (2003), 77-161.

[I] H. Iritani, Real and integral structures in quantum cohomology I: toric orbifolds, arXiv:0712.2204.

[K-S-T 1] H. Kajiura, K. Saito and A. Takahashi, Matrix Factorizations and Representations of Quivers II: type ADE case, Adv. Math. 211 (2007), no. 1, 327-362.

[K-S-T 2] _ , Triangulated categories of matrix factorizations for regular systems of weights with $\epsilon=-1$, arXiv:0708.0210.

[K-S-T 3] _ Weighted projective lines associated to regular systems of weights, in preparation.

[Ka-Ko-P] L. Katzarkov, M. Kontsevich, T. Pantev, Calculating Gromov-Witten invariants from Fukaya category, in preparation.

[Ko] M. Kontsevich, Homological algebra of mirror symmetry, Proceedings of ICM (Zurich,1994), 120139, Basel: Birkháuser (1995).

[Ko-So] M. Kontsevich and Soibelmann, Notes on $\mathcal{A}_{\infty}$-algebras, $\mathcal{A}_{\infty}$-categories and non-commutative geometry. I, arXiv:math/0606241.

[Ma1] Y. Manin, Frobenius manifolds, quantum cohomology, and moduli spaces, American Math.Society Colloquium Publ. v.47, 1999. 
[Mi] J. Milnor, Singular points of complex hypersurfaces, Ann. of Math. Studies, vol. 61, Princeton Univ. Press, 1968.

[Sab] C. Sabbah, Déformations isomonodromiques et variétés de Frobenius, 2002, EDP Sciences.

[S1] K. Saito, On a linear Structure of the Quotient variety by a Finite Reflection group, RIMS-288, Kyoto Univ., June 1979.

[S2] - On the periods of primitive integrals I, preprint Harvard (1980).

[S3] , Period Mapping Associated to a Primitive Form, Publ. RIMS, Kyoto University 19 (1983), $1231-1264$.

[S4] - Primitive forms for a universal unfolding of a function with an isolated critical point, J. Fac. Sci. Univ. Tokyo Sect. IA 28 (1982), .

[S5] - The higher residue pairings $K_{F}^{(k)}$ for a family of hypersurface singular points, Proceedings of Symposia in Pure Mathematics Vol. 40 (1983), part 2, pp. 441-463.

[S6] _ Primitive Automorphic Forms, Mathematics Unlimited - 2001 and Beyond, edited by Engquist \& Schmidt, Springer Verlag, (2001) 1003-1018.

[S7] Uniformization of the Orbifold of a Finite Reflection Group Uniformization of the orbifold of a finite reflection group, in Frobenius Manifolds, C. Hertling, M. Marcolli (eds.), Aspect Math. E36 (2004), 265-320.

[S8] - Towards a categorical construction of Lie algebras, Advanced Studies in Pure Mathematics 50, (2008), Algebraic Geometry in east Asia-Hanoi 2005, 101-175.

[S9] — Extended affine root systems, II. (Flat invariansts), Publ.RIMS 26 (1990) no.2, 15-78.

[S10] _ On a linear Structure of the Quotient Variety by a Finite Reflection Group, Publ.RIMS 29 (1993),535-579.

[SM] M. Saito, On the structure of Brieskorn lattice, Ann. Inst, Fourier (Grenoble) 39 (1989), no.1, $27-72$.

[S-Y-S] K. Saito, T. Yano and J. Sekiguchi, On a certaingenerator system of the ring of invariants of a finite reflection group, Commun. in Alg., 8(4) (1980), 373-408.

[Sat] I. Satake, Flat Structure for the simple Elliptic Singularities of type $\widetilde{E}_{6}$, Proceedings of 37 th Taniguchi Symposium, Birkhäuser, 1998.

[T1] A. Takahashi, Matrix Factorizations and Representations of Quivers I, math.AG/0506347.

[T2] — Weighted Projective Lines Associated to Regular Systems of Weights of dual Type, arXiv:0711.3907.

[Th] R. Thom, Structural stability and morphogenesis. Translated from the French by D. H. Fowler. With a foreword by C. H. Waddington. Second printing. W. A. Benjamin, Inc., Reading, Mass.London-Amsterdam, 1976.

RIMS, KYoto UnIVERSITY, KYOTO 606-8502, JAPAN

E-mail address: saito@kurims.kyoto-u.ac.jp

Department of Mathematics, Graduate School of Science, Osaka University, ToyONAKA OSAKA, 560-0043, JAPAN

E-mail address: takahashi@math.sci.osaka-u.ac.jp 